\title{
ORGANIZAÇÕES E PESSOAS PARTICIPANTES DO CICLO DE PREMIAÇÃO 2004
}

Encerrado o período de inscrições para o Ciclo de Premiação 2004 do Programa GESTÃO PÚBLICA E CIDADANIA, 1.191 programas, projetos ou atividades haviam enviado material e estavam aptos a participar do processo seletivo para a fase subsequiente.

A partir daí, a análise do material mobilizou uma Comissão de Seleção formada por membros de organizações da sociedade civil e pesquisadores de universidades e de instituições de pesquisa, todos eles de alguma forma atuantes na busca da melhoria da administração pública brasileira. Subdividido em grupos de quatro, esta Comissão organizou-se de forma a garantir que cada iniciativa governamental inscrita fosse analisada e discutida, preliminarmente, por dois grupos diferentes. A partir daí, uma nova rodada de discussão geral, na qual cada caso foi exaustivamente debatido, permitiu que se chegasse à indicação dos semifinalistas com base na construção gradual de consensos.

Uma vez indicados os 100 semifinalistas, foi-lhes solicitado responder a um questionáriopadrão, composto de 18 perguntas (ver quadro III, p.31 e 32). Estas respostas subsidiaram a análise para indicação dos 30 pré-finalistas, em processo idêntico ao da etapa anterior. Escolhidas estas 30 iniciativas, um conjunto de especialistas e estudiosos foi a campo conhecer as experiências in loco, preparando então os "Relatórios de Visita de Campo". Estes relatórios possibilitaram a escolha dos 20 finalistas, em uma nova bateria de discussões. Todo o material recolhido e produzido ao longo deste processo passou a compor o Banco de Dados do Programa, estando disponível a qualquer interessado para consulta e obtenção de cópias.

O Ciclo de Premiação 2004 encerra-se com um evento realizado na sede do Banco Nacional de Desenvolvimento Econômico e Social - BNDES, no Rio de Janeiro, no qual os responsáveis pelas 20 iniciativas finalistas apresentam ao público presente e debatem com uma Banca Julgadora os programas desenvolvidos pelas organizações públicas das quais fazem parte. Esta Banca reúne-se ao final do dia e indica cinco destaques.

A seguir, apresentamos as organizações e pessoas que de forma mais ativa estiveram envolvidas em cada uma das etapas deste esforço coletivo para identificar e disseminar os caminhos que vêm sendo percorridos rumo a uma melhor administração pública. 


\section{EQUIPE DO PROGRAMA GESTÃO PÚBLICA E CIDADANIA}

Coordenação Acadêmica

Coordenadora Administrativa

Secretária do Programa

Assistente Administrativa

Pesquisadores

Disseminação

Monitores Acadêmicos
Peter Spink (Diretor)

Marta Ferreira Santos Farah (Vice-Diretora)

Fernando Guilherme Tenório

Ilka Camarotti

Ricardo Bresler

Fabiana Paschoal Sanches de Moura

Marlei de Oliveira

Rosa Maria de Lima e Silva

Cibele Franzese

Fernanda Martinez de Oliveira

Jacqueline Isaac Machado Brigagão

Janaína Valéria Mattos

Lília Asuca Sumiya

Marco Antonio Carvalho Teixeira

Otávio Prado

Paula Maciel Pedroti

Silvia Craveiro

Carla Coelho Ribeiro

Hélio Batista Barboza

Estêvão Passos Eller

Nathalie Perret

Melissa Godoy

Daniela P. Sanches 


\section{COMITÊ TÉCNICO}

Beto Ricardo

Edna Ramos de Castro

Eliana Maria Custódio

Humberto Marques Filho

José Antônio Gomes de Pinho

Laura da Veiga

Luis Roque Klering

Marlene Libardoni

Nancy Cárdia

Paulo Pankararu

Pedro Jacobi

Silvio Caccia Bava
- Instituto Socioambiental (ISA)

Núcleo de Altos Estudos Amazônicos - Universidade Federal do Pará

- GELEDES - Instituto da Mulher Negra

Universidade Federal do Paraíba - Programa de Pós-Graduação - em Administração

Universidade Federal da Bahia - Núcleo de Pesquisa e PósGraduação

Programa de Mestrado em Administração Pública - Escola de - Governo de Minas Gerais / Fundação João Pinheiro

Universidade Federal do Rio Grande do Sul Programa de Pós- Graduação em Administração

- AGENDE - Ações em Gênero, Cidadania e Desenvolvimento

- Núcleo de Estudos da Violência - USP

Coordenação das Organizações Indígenas da Amazônia

- Brasileira (COIAB)

Programa de Pós-Graduação em Ciência Ambiental da

- Universidade de São Paulo (Procam/USP)

PÓLIS - Instituto de Estudos, Formação e Assistência em

- Políticas Sociais 
CADERNOS GESTÃO PÚBLICA E CIDADANIA

\section{COMISSÃO DE SELEÇÃO - 1 FASE (INDICAÇÃO DOS SEMIFINALISTAS)}

Alexandre Alves Schneider

Ana Maria Wilheim

Ana Paula Macedo Soares

Angela Regina Pires Macedo

Antonio Faria

Antonio Olenscki

Antonio Sérgio Fernandes

Carla Coelho

Cecília Olivieri

Cibele Franzese

Daniela Azevedo

Diego Pedalino

Edna Castro

Eduardo Caldas

Eliana Custódio

Estêvão Eller

Fabio Storino

Fernanda Oliveira

Fernanda Teles Lima

Fernando Machado

Fernando Monteiro

Fernando Nogueira

Frances Barreto Jones

Francine Lemos
- Mestrado em Administração Pública e Governo - EAESP-FGV

- Fundação Abring

- Instituto Votorantim

- Banco Nacional de Desenvolvimento Econômico e Social BNDES

- Mestre em Administração Pública e Governo EAESP-FGV

- Doutorado em Administração Pública e Governo EAESP-FGV

- Professor da UNESP - Campus Araraquara

- Gestão Pública e Cidadania

- Doutorado em Administração Pública e Governo EAESP-FGV

- Gestão Pública e Cidadania

- Mestrado em Administração de Empresas EAESP-FGV

- Graduação em Administração de Empresas EAESP-FGV

- Núcleo de Altos Estudos Amazônicos - Universidade Federal do Pará

- Instituto de Estudo, Formação e Assessoria em Políticas Sociais - PÓLIS

- Instituto da Mulher Negra - GELEDES

- Gestão Pública e Cidadania

- Doutorado em Administração Pública e Governo - EAESPFGV

- Gestão Pública e Cidadania

- Mestrado em Administração Pública e Governo - EAESP-FGV

- Mestre em Administração Pública e Governo - EAESP-FGV

- Programa de Pós-Graduação em Ciência Ambiental da Universidade de São Paulo - Procam/USP

- Mestrado em Administração Pública e Governo EAESP-FGV

- Jornalista Agência Reuters

- Graduação em Administração Pública e Governo - EAESPFGV 


\section{Gabriela Lotta}

Guilherme de Paula e Silva

Heitor Battaglia

Hélio Barboza

Hironobu Sano

Humberto Marques

Ilka Camarotti

Jacqueline Brigagão

Janaína Mattos

José Agnaldo Gomes

José Luiz Dutra

José Antônio Pinho

Juliana Simões Speranza

Lilia Asuca Sumiya

Luciano Bueno

Luis Roque Klering

Márcia Vasconcellos

Marcio Wegmann

Maria Guadalupe Rios

Mário Aquino

Marisa Tardelli de Azevedo

Mary Cheng

Melissa de Godoy

Melissa Rizzo Battistella

Nathalie Perret

Otávio Prado
- Instituto de Estudo, Formação e Assessoria em Políticas Sociais - PÓLIS

- Mestre em Administração Pública e Governo - EAESP-FGV

- Doutor em Psicologia Social - PUC/SP

- Gestão Pública e Cidadania

- Doutorado em Administração Pública e Governo - EAESPFGV

- Programa de Pós-Graduação em Administração Universidade Federal da Paraíba

- Gestão Pública e Cidadania

- Gestão Pública e Cidadania

- Gestão Pública e Cidadania

- Mestrado em Psicologia Social PUC

- EBAPE/FGV

- Núcleo de Pesquisa e Pós-Graduação em Administração Universidade Federal da Bahia

- Mestrado em Desenvolvimento, Agricultura e Sociedade na Universidade Federal Rural do Rio de Janeiro - UFRRJ

- Gestão Pública e Cidadania

- Doutorado em Administração Pública e Governo - EAESPFGV

- Programa de Pós Graduação em Administração - Universidade Federal do Rio Grande do Sul

- Ações em Gênero Cidadania e Desenvolvimento - AGENDE

- Banco Nacional de Desenvolvimento Econômico e Social BNDES

- Universidade de Austin - Texas/EUA

- Professor EAESP-FGV

- Fundação Abrinq

- Graduada em Administração de Empresas EAESP-FGV

- Gestão Pública e Cidadania

- Instituto Votorantim

- Gestão Pública e Cidadania

- Gestão Pública e Cidadania 
Pedro Jacobi

Pedro Paulo Piani

Rafael D'Almeida Martins

Ricardo Bresler

Roberta Clemente

Rosana Paula Orlando

Sandra Wegmann

Sérgio de Arruda Sampaio

Silvia Craveiro

Silvia Kawata

Silvio Caccia Bava

Thiago Cacique

Veronika Paulics
- Programa de Pós-Graduação em Ciência Ambiental da Universidade de São Paulo(Procam/USP)

- Doutorado em Psicologia Social - PUC/SP

- Instituto de Estudo, Formação e Assessoria em Políticas Sociais - PÓLIS

- Professor da EAESP-FGV

- Doutorado em Administração Pública e Governo - EAESPFGV

- Fundação Abrinq

- Universidade de Austin - Texas/EUA

- Mestrado em Administração Pública e Governo - EAESP-FGV

- Gestão Pública e Cidadania

- Mestre em Administração Pública e Governo - EAESP-FGV

- Instituto de Estudo, Formação e Assessoria em Políticas Sociais - PÓLIS

- Mestrado em Administração Pública e Governo - EAESP-FGV

- Instituto de Estudo, Formação e Assessoria em Políticas Sociais - PÓLIS 


\section{COMISSÃO DE SELEÇÃO - 2a FASE (INDICAÇÃO DOS PRÉ-FINALISTAS)}

\begin{tabular}{|c|c|}
\hline Ana Maria Wilheim & - Fundação Abrinq \\
\hline Antônio José Faria da Costa & - Mestrado em Administração Pública e Governo - EAESP-FGV \\
\hline Antônio Sérgio Fernandes & - Professor da UNESP - Campus Araraquara \\
\hline Bruno L. D.Costa & - Escola de Governo / Fundação João Pinheiro \\
\hline Cristina Neme & - Núcleo de Estudos da Violência - USP \\
\hline Djair Pichiari & - Professor da Fundação Getúlio Vargas EAESP-FGV \\
\hline Eduardo Caldas & - Mestrado em Administração Pública e Governo - EAESP-FGV \\
\hline Eliana M. Custódio & - Instituto da Mulher Negra - GELEDES \\
\hline Fernando Tenório & - Professor da Fundação Getúlio Vargas - EBAPE-FGV \\
\hline Ideli Domingues & - Professora da Fundação Getúlio Vargas - EAESP-FGV \\
\hline Ilka Camarotti & - Projeto Práticas Públicas e Pobreza - Gestão Pública e Cidadania \\
\hline Ivan Prado & - Friedrich Ebert Stiftung - ILDES \\
\hline Jacqueline Brigagão & - Gestão Pública e Cidadania \\
\hline José Antônio Pinho & $\begin{array}{l}\text { Núcleo de Pesquisa e Pós-Graduação em Administração - } \\
\text { Universidade Federal da Bahia }\end{array}$ \\
\hline Lúcia Calil & - Sal da Terra \\
\hline Luis Roque Klering & $\begin{array}{l}\text { Programa de Pós-Graduação em Administração - Universidade } \\
\text { - Federal do Rio Grande do Sul }\end{array}$ \\
\hline Marco Antonio C. Teixeira & - Gestão Pública e Cidadania \\
\hline $\begin{array}{l}\text { Maria do Carmo M. Toledo } \\
\text { Cruz }\end{array}$ & - Fundação Prefeito Faria Lima - CEPAM/USP \\
\hline Marilena Jamur & - Programa de Pós-Graduação em Serviço Social da Puc- Rio \\
\hline Pedro Jacobi & $\begin{array}{l}\text { Programa de Pós-Graduação em Ciência Ambiental da } \\
\text { - Universidade de São Paulo (Procam / USP) }\end{array}$ \\
\hline Ricardo Bresler & - Professor da Fundação Getúlio Vargas - EAESP-FGV \\
\hline Ricardo Bueno & - Mestrado em Administração Pública e Governo - EAESP-FGV \\
\hline Silvia Salgado & - Fundação Prefeito Faria Lima - CEPAM/USP \\
\hline Verônika Paulics & $\begin{array}{l}\text { PÓLIS - Instituto de Estudos, Formação e Assessoria em Políticas } \\
\text { Sociais }\end{array}$ \\
\hline
\end{tabular}




\title{
COMISSÃO DE SELEÇÃO - $3^{\text {a }}$ FASE (INDICAÇÃO DOS FINALISTAS)
}

\author{
Cristina Neme \\ Edna de Castro \\ Eliana Maria das Graças \\ Custódio \\ Fernando Guilherme \\ Tenório \\ Humberto Marques Filho \\ Ilka Camarotti \\ José Antônio Pinho \\ Laura da Veiga \\ Luis Roque Klering \\ Marlene Libardoni \\ Pedro Jacobi \\ Verônika Paulics \\ - Núcleo de Estudos da Violência - USP \\ Núcleo de Altos Estudos Amazônicos - Universidade Federal do \\ Pará \\ - Instituto da Mulher Negra - GELEDES \\ - Fundação Getúlio Vargas EBAPE - FGV \\ Programa de Pós-Graduação em Administração - Universidade \\ Federal da Paraíba \\ - Projeto Práticas Públicas e Pobreza - Gestão Pública e Cidadania \\ Núcleo de Pesquisa e Pós-Graduação em Administração - \\ Universidade Federal da Bahia \\ Programa de Mestrado em Administração Pública Fundação \\ Escola de Governo / Fundação João Pinheiro Minas Gerais \\ Programa de Pós-Graduação em Administração - Universidade \\ Federal do Rio Grande do Sul \\ - Ações em Gênero Cidadania e Desenvolvimento - AGENDE \\ Programa de Pós-Graduação em Ciência Ambiental da \\ Universidade de São Paulo (Procam / USP) \\ Instituto de Estudos, Formação e Assessoria em Políticas Sociais \\ - PÓLIS
}




\section{VISITAS DE CAMPO}

Ação Comunitária em Arte e Ofício- Experiências Educativas em Comunidades Rurais Negras no Pará - Pará

Técnico: Antônio Faria (Mestre em Administração Pública e Governo EAESP-FGV)

“Água para Todos" - Preservar Nossos Recursos Hídricos com Qualidade, Quantidade e Responsabilidade - Japonvar - MG

Técnica: Maria Castellano (Programa de Pós-Graduação em Ciência Ambiental da Universidade de São Paulo - Procam / USP)

Beagalê - Belo Horizonte - $\mathrm{MG}$

Técnico: Heitor Bataggia (Doutor em Psicologia Social - PUC/SP)

Cheque Moradia - Goiás

Técnico: Guilherme Henrique de Paula e Silva (Mestre em Administração Pública e Governo EAESP-FGV)

Coleta Seletiva Londrina "Reciclando Vidas" - Londrina - PR

Técnica: Gina Rizpah Besem (Faculdade de Educação Ambiental - SENAC/ SP)

Construindo e Fortalecendo Capacidades Sociais para Discutir e Planejar a Cidade: A Experiência de Elaboração do Plano Diretor Participativo de Santo André - Santo André - SP Técnicos: Marco Antônio C. Teixeira (Gestão Pública e Cidadania) e Sandra Wegmann (Universidade de Austin - Texas)

Desenvolvimento Florestal Sustentável na Amazônia Brasileira - Acre Técnicos: Luis Mario Fujiwara (Doutorado - Universidade de Austin- Texas) e Estêvão Passos Eller (Gestão Pública e Cidadania)

Educação para a Convivência com o Semi-Árido - Juazeiro - BA

Técnica: Maria do Carmo M. T. Cruz (Fundação Prefeito Faria Lima - CEPAM/USP)

Escola do Campo - Araraquara - SP

Técnicos: Hironobu Sano (Doutorado em Administração Pública e Governo - EAESP-FGV) e Juliana Speranza- (Mestrado em Desenvolvimento, Agricultura e Sociedade - UFRRJ)

Escola Profissionalizante Raimunda da Silva Soares - Belo Horizonte - MG Técnica: Lilia Asuca Sumiya (Gestão Pública e Cidadania) e Maria Fernanda Alessio (Graduanda em Administração Pública EAESP- FGV)

Formação Intercultural Diferenciada e Bilíngüe de Professores Indígenas - Acre Técnica: Silvia Craveiro (Gestão Pública e Cidadania) Nathalie Perret Gentil (Gestão Pública e Cidadania) 
Fortalecimento da ADMOC- Agência de Desenvolvimento do Meio Oeste Catarinense Santa Catarina

Técnica: Cibele Franzese (Gestão Pública e Cidadania)

Fossa Ecológica - Sobral - CE

Técnico: Ricardo Bueno (Mestre em Administração Pública e Governo - EAESP- FGV)

Geração de Trabalho e Renda - Chapecó - SC

Técnica: Fernanda Teles de Lima (Mestrado em Administração Pública e Governo - EAESP-FGV)

Legislativo Interativo e Participativo - Santa Terezinha - MT

Técnico: Rafael D’Almeida Martins (Instituto de Estudos, Formação e Assessoria em Políticas Sociais - PÓLIS)

Lei Municipal de Tombamento - Proteção do Patrimônio Cultural e Natural do Município de Dois Irmãos e Criação do Conselho Municipal do Patrimônio Cultural - Dois Irmãos - RS Técnico: Antônio Sérgio de Araújo Fernandes (UNESP - Campus Araraquara)

Masculinidade e Cidadania - Diadema - SP .

Técnicas: Janaína Mattos (Gestão Pública e Cidadania) e Mary Cheng (Graduada em Administração de Empresas EAESP-FGV)

Moradia Indígena - São Sebastião - SP

Técnicos: Antônio Olenscki (Doutorado em Administração Pública e Governo - EAESP-FGV) e Melissa Godoy (Gestão Pública e Cidadania)

Oficina Boracéa - São Paulo - SP

Técnica: Janaína Simões (Mestranda em Administração - EBAPE- FGV)

Organização Sócio-Política do Povo Xukuru do Ororubá - Povo Xukuru - Pesqueira - PE Técnico: Pedro Paulo Piani (Doutorado em Psicologia Social - PUC/SP)

Produção Limpa - Guaporé- RS

Técnico: Fernando Monteiro (Programa de Pós-Graduação em Ciência Ambiental da Universidade de São Paulo - Procam / USP)

PROCAP - Programa Municipal de Apoio à Cadeia Produtiva da Caprinovinocultura Mossoró - RN

Técnico: Fernando Nogueira (Mestrado em Administração Pública e Governo - EAESP-FGV)

Programa Estadual de Prevenção e Combate à Violência Contra a Mulher - Mato Grosso do Sul

Técnica: Rita de Cássia Bento Barboza (Assistente Social)

Projeto Crescer - Boa Vista - RR

Técnica: Silvia Salgado (Fundação Prefeito Faria Lima - CEPAM/USP) 
Piraí: Município Digital - Piraí - RJ

Técnico: Edson Sadao Izuka (Doutorado em Administração Pública e Governo - EAESP-FGV)

Recife sem Palafitas - Recife - PE

Técnica: Fernanda Oliveira (Gestão Pública e Cidadania)

Segurança Contra Incêndio em Assentamentos Urbanos Precários - São Paulo

Técnicos: Ricardo Bresler (Professor da FGV- EAESP) e Carla Coelho (Gestão Pública e Cidadania)

SESAMAR / CAPS / Araçuaí - Serviço de Saúde Mental / Centro de Atenção Psicossocial de Araçuaí - Araçuaí - MG

Técnica: Jacqueline I. Machado Brigagão (Gestão Pública e Cidadania)

Tejubode: Mais que Uma Festa, Uma Idéia de Desenvolvimento - Tejuçuoca - CE Técnica: Gabriela Lotta (Instituto de Estudos, Formação e Assessoria em Políticas Sociais PÓLIS)

Turismo como Alternativa de Desenvolvimento no Semi-Árido - Cabaceiras - PB

Técnico: José Luiz Dutra (Mestre em Administração EBAPE- FGV) 
FINALISTAS 2004

\begin{tabular}{|c|c|}
\hline Nome do Programa & Pessoa Responsável \\
\hline Coleta Seletiva Londrina "Reciclando Vidas" & - Rosimeire Suzuki \\
\hline Organização Sócio- Política do Povo Xukuru do Ororubá & $\begin{array}{l}\text { Marcos Luidison de } \\
\text { Araújo }\end{array}$ \\
\hline Escola do Campo & $\begin{array}{l}\text { Alexandre Luis Martins } \\
\text { de Freitas }\end{array}$ \\
\hline Masculinidade e Cidadania & $\begin{array}{l}\text { Analdeci Moreira dos } \\
\text { Santos }\end{array}$ \\
\hline $\begin{array}{l}\text { Ação Comunitária em Arte e Ofício- Experiências Educativas } \\
\text { em Comunidades Rurais Negras no Pará }\end{array}$ & $\begin{array}{l}\text { Dina Maria César de } \\
\text { Oliveira }\end{array}$ \\
\hline $\begin{array}{l}\text { PROCAP- Programa Municipal de Apoio à Cadeia Produtiva } \\
\text { da Caprinovinocultura }\end{array}$ & $\begin{array}{l}\text { Antonio Gilberto de } \\
\text { Oliveira Jales }\end{array}$ \\
\hline $\begin{array}{l}\text { Lei Municipal de Tombamento - Proteção do Patrimônio } \\
\text { Cultural e Natural do Município de Dois Irmãos e Criação do } \\
\text { Conselho Municipal do Patrimônio Cultural }\end{array}$ & - Juarez Stein \\
\hline SESAMAR/ CAPS/ Araçuaí & $\begin{array}{l}\text { Maria do Carmo Ferreira } \\
\text { da Silva (Cacá) }\end{array}$ \\
\hline Cheque Moradia & - Álvaro César Lourenço \\
\hline $\begin{array}{l}\text { Programa Estadual de Prevenção e Combate à Violência Contra } \\
\text { a Mulher }\end{array}$ & - Sandra Regina Alt \\
\hline Escola Profissionalizante Raimunda da Silva Soares & - Vânia Diniz \\
\hline Piraí: Município Digital & - Franklin Dias Coelho \\
\hline $\begin{array}{l}\text { Formação Intercultural Diferenciada e Bilíngüe de Professores } \\
\text { Indígenas }\end{array}$ & $\begin{array}{l}\text { Manoel Estébio } \\
\text { Cavalcante da Cunha }\end{array}$ \\
\hline Produção Limpa & - Denise Bordin Roos \\
\hline Geração de Trabalho e Renda & - Iraci de Andrade \\
\hline Projeto Crescer & - Cícero Campelo Neto \\
\hline Turismo como Alternativa de Desenvolvimento no Semi-Árido & $\begin{array}{l}\text { - Márcia Cristina de Souza } \\
\text { Truta }\end{array}$ \\
\hline Oficina Boracéa & - Aldaíza Sposati \\
\hline Legislativo Interativo e Participativo & $\begin{array}{l}\text { Dagmar Aparecida } \\
\text { Teodoro Gatti }\end{array}$ \\
\hline Desenvolvimento Florestal Sustentável na Amazônia Brasileira & - João César Dotto \\
\hline
\end{tabular}

